\begin{tabular}{|c|l|}
\hline Title & Control of a two-dimensional molecular structure by cooperative hal ogen and hydrogen bonds \\
\hline Author(s) & Y asuda, Satoshi; Furuya, A tom; Murakoshi, Kei \\
\hline Citation & $\begin{array}{l}\text { RSC advances, 4(102), 58567-58572 } \\
\text { https://doi.org/40.1039/c4ra10235e }\end{array}$ \\
\hline Issue Date & 2014_10-30 \\
\hline Doc URL & http://hdl.handle.net/2115/60148 \\
\hline Type & article(author version) \\
\hline File Information & RSC_adv_2014_58567-.pdf \\
\hline
\end{tabular}

Instructions for use 


\title{
ARTICLE
}

\section{Control of Two-Dimensional Molecular Structure by Cooperative Halogen and Hydrogen Bonds}

\author{
Satoshi Yasuda, ${ }^{* a, b}$ Atom Furuya ${ }^{a}$ and Kei Murakoshi* ${ }^{a}$
}

Received ooth January 2012, Accepted ooth January 2012

DOI: 10.1039/xoxxooooox

www.rsc.org/
The cooperative effect of hydrogen and halogen bonds on the two-dimensional (2D) molecular arrangement on highly oriented pyrolytic graphite (HOPG) was studied by scanning tunneling microscopy. The terephthalic acid (TPA) molecule, which has two carboxyl groups attached at the para positions of a benzene ring, formed a one-dimensional (1D) linear non-covalent network structure on HOPG by hydrogen bonds between the carboxyl groups of neighboring molecules. However, unlike the TPA molecule, Br substituted TPA molecules were found to form different non-covalent network structures. Owing to $\mathrm{Br}^{\cdots} \mathrm{O}$ halogen and hydrogen bonds, bromo-substituted TPA (2-bromoterephthalic acid) formed a 1D ladder-like non-covalent network structure, whereas dibromo-substituted TPA (2,5-dibromoterephthalic acid) formed a $2 \mathrm{D}$ non-covalent lattice network on HOPG. These results strongly indicate that $\mathrm{Br} \cdots \mathrm{O}$ halogen significantly contribute to determine the molecular assembly as well as hydrogen bonds for molecules containing bromine groups and hydrogen bonding groups. These results provided deep fundamental insight into the cooperative effect of halogen and hydrogen bonds in 2D molecular assembly. In addition, we have demonstrated that these interactive bonds are promising for the precise design of 2D molecular architectures.

\section{Introduction}

Fabrication of new two-dimensional (2D) materials using molecular self-assembly on substrate surfaces has attracted attention for future applications such as ultra-thin electronic and optoelectronic devices. ${ }^{1}$ In general, molecular self-assembly on surfaces is governed by the balance between moleculesubstrate and molecule-molecule interactions. In particular, in the case of weak molecule-substrate interactions, non-covalent molecule-molecule interactions become dominant for selfassembly. One of the key interactions for forming well-ordered 2D molecular structures is the hydrogen bond, because of not only its strong non-covalent interactions but also its selective and directional nature, unlike van der Waals interactions. ${ }^{2-4}$ For example, the terephthalic acid (TPA) molecule, which has two carboxyl groups at the para positions of a benzene ring, forms self-assembled monolayers on highly oriented pyrolytic graphite (HOPG). ${ }^{2,3}$ Because of the weak molecule-substrate interaction, the TPA molecules assemble in a well-defined 2D molecular structure with a hydrogen bonded network between the carboxyl groups. Based on the hydrogen bond, the various non-covalent 2D molecular structures such as porous honeycombs, kagome, twin rows, rectangles and clips have been widely studied. ${ }^{1-8}$

Recently, to further control 2D molecular structures, the simultaneous use of not only hydrogen bonds but also other non-covalent interactions has become an intriguing research topic. Currently, as another candidate molecule-molecule interaction, the halogen bond has attracted significantly attention. The halogen bond is a directional non-covalent

interaction involving halogen atoms and steams from the anisotropic distribution of the electron of the halogen atoms. ${ }^{9-12}$ Typical bonding energies of hydrogen bonds are from 1 to 167 $\mathrm{kJ} / \mathrm{mol}$ depending on their environment, ${ }^{15}$ while halogen bonds are from 6 to $180 \mathrm{~kJ} / \mathrm{mol} .^{9-14}$ Although typical halogen bonds are relatively weak compared with hydrogen bonds, it has shown that, in some cases, the strength of halogen bonds can surpass classical hydrogen bonds, resulting in halogen bonds being the dominant driving force in the three-dimensional crystallization of organic molecules and the association of biological molecules. ${ }^{16,17}$ From this viewpoint, using halogen bonds for assembling 2D molecular structures has advanced in recent years. For example, there have been studies on the effect of the halogen bond on 2D self-assembled monolayers, showing that halogen bonds, such as $\mathrm{X}^{\cdots} \mathrm{X}, \mathrm{X}{ }^{\cdots}$ aryl and $\mathrm{X}{ }^{\cdots} \mathrm{H}-\mathrm{C}$ (X: halogen atoms), behave as the primary interactions that control molecular assembly. ${ }^{18-26}$ However, although research related to $2 \mathrm{D}$ molecular self-assembly involving either hydrogen or halogen bonds has already been reported, there have been few experimental studies on 2D molecular selfassemblies formed by both hydrogen and halogen bonds. It is valuable for obtaining the knowledge in the viewpoint of not only fundamental understanding of liquid-solid interface but also further development of 2D molecular assembly. In this study, we investigate the influence of the simultaneous use of hydrogen and halogen bonds on 2D molecular structures using scanning tunnelling microscopy (STM).

\section{Experimental}




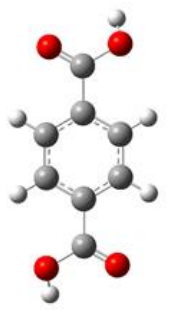

TPA

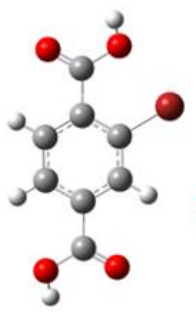

BTPA

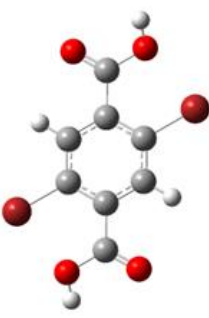

DBTPA
Figure 1. Molecular structures of terephthalic acid (TPA), 2bromoterephthalic acid (BTPA), and 2,5-dibromoterephthalic acid (DBTPA). Carbon: gray, oxygen: red, bromine: brown, and hydrogen: white.

The molecular structures of TPA, 2-bromoterephthalic acid (BTPA), and 2,5-dibromoterephthalic acid (DBTPA) are shown in Fig. 1. All the molecules have two carboxyl groups, while BTPA and DBTPA are modified with one and two bromo groups, respectively. These molecules were dissolved in heptanoic acid, which is an insulator with low vapor pressure and consequently enables stable and prolonged STM measurements. A droplet of the saturated solution $(\sim 100 \mu \mathrm{L})$ was deposited on freshly cleaved HOPG (grade ZYB; advanced Ceramics Inc., Cleveland, OH, USA). STM investigations were performed with a Nanoscope IIIa scanning probe microscope system (Bruker, Billerica, USA) in aqueous conditions. All STM images were obtained in constant height mode using a mechanically-cut Pt/Ir tip. To assign the origin of the molecular packing structure, the STM images were compared with those of molecular orbital and electrostatic potential calculations. These calculations for all the molecules were performed using density functional theory with the B3LYP functional and 6-31G (d,p) set in Gaussian 09.

\section{Results and discussion}

Figure 2(a)-(c) shows large-scale STM images of selfassembled TPA, BTPA, and DBTPA monolayers on HOPG. In the case of TPA, homogeneous monolayer formation was observed over all of the surface (Fig. 2(a)), which is good agreement with previous results. ${ }^{6}$ In contrast to the TPA monolayer, both BTPA and DBTPA formed inhomogeneous monolayers consisting of several small domains (Figs. 2(b) and (c)). These differences were not surprising given the differences of the intermolecular interactions in the monolayers.

To analyze the intermolecular interactions in detail, the
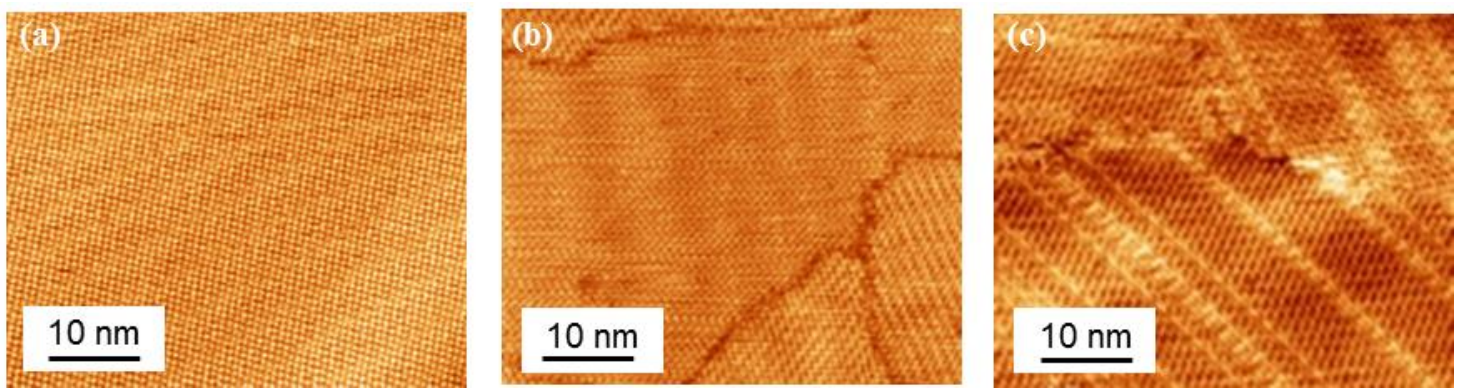

Figure 2. Large scale STM images of self-assembled (a) TPA $\left(V_{\mathrm{s}}=-0.5 \mathrm{~V}, I_{\mathrm{t}}=0.1 \mathrm{nA}\right)$, (b) BTPA( $\left(V_{\mathrm{s}}=-0.5 \mathrm{~V}, I_{\mathrm{t}}=0.1 \mathrm{nA}\right)$, and $(\mathrm{c})$ $\operatorname{DBTPA}\left(V_{\mathrm{s}}=-0.5 \mathrm{~V}, I_{\mathrm{t}}=0.1 \mathrm{nA}\right)$. 


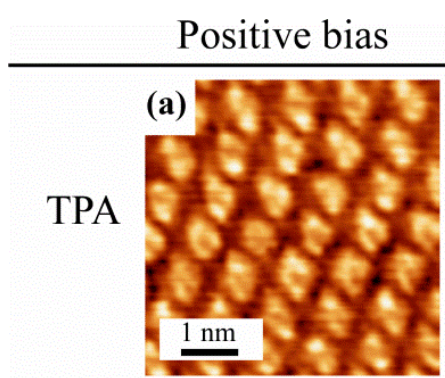

Negative bias
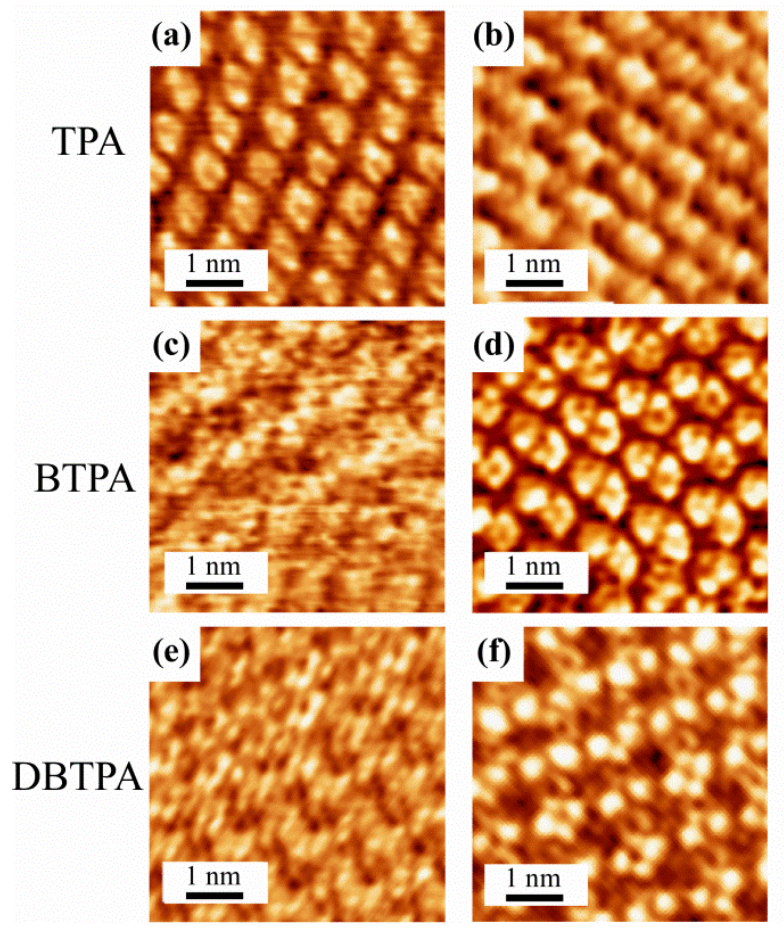

Figure 3. Bias-dependent STM images. (a), (c) and (e) are positive sample bias STM images of TPA, BTPA and DBTPA $\left(V_{\mathrm{s}}=+0.5 \mathrm{~V}, I_{\mathrm{t}}=0.20 \mathrm{nA}\right)$. (b), (d) and (f) are negative sample bias STM images of TPA, BTPA and DBTPA $\left(V_{\mathrm{s}}=-\right.$ $\left.0.5 \mathrm{~V}, I_{\mathrm{t}}=0.20 \mathrm{nA}\right)$.

relatively bright spot in the STM images with negative sample bias (HOMO state), suggesting an enhancement in the tunneling current through the HOMO state of the halogen groups. ${ }^{27}$ The mechanism has been proposed that high atomic polarizability of halogen atom reduces the substrate work function and energy level of the HOMOs by weak coupling of orbitals, leading to an increase of resonance tunneling current. $^{25-27}$ From these results, we can conclude that the observed relatively bright spots adjacent to the benzene rings in STM images are definitely bromo groups of BTPA and DBTPA. On the other hand, unclear STM images were acquired at positive sample bias (LUMO state). The reason for this is still unclear, and further investigation is being undertaken.

Based on the interpretation of the bias dependent STM images and molecular orbital calculations, proposed molecular packing structures are given in Fig. 5, and the lattice parameters are summarized in Table 1. For the TPA monolayer, owing to two hydrogen bonds between adjacent carboxyl groups, the TPA molecules form a one-dimensional (1D) linear structure, which is good agreement with previous results. ${ }^{2}$ Since carboxyl groups can act as both a donor and acceptor for hydrogen bonds, and hydrogen bonds are stronger than van der Waals forces, formation of a 1D non-covalent network becomes possible (Figs. 5(a) and (d)). Such structures assemble in parallel to each other because of van der Waals forces between adjacent structures, and a stable homogeneous monolayer can be formed in a seamless manner, as shown Figs. 2(a) and (b).

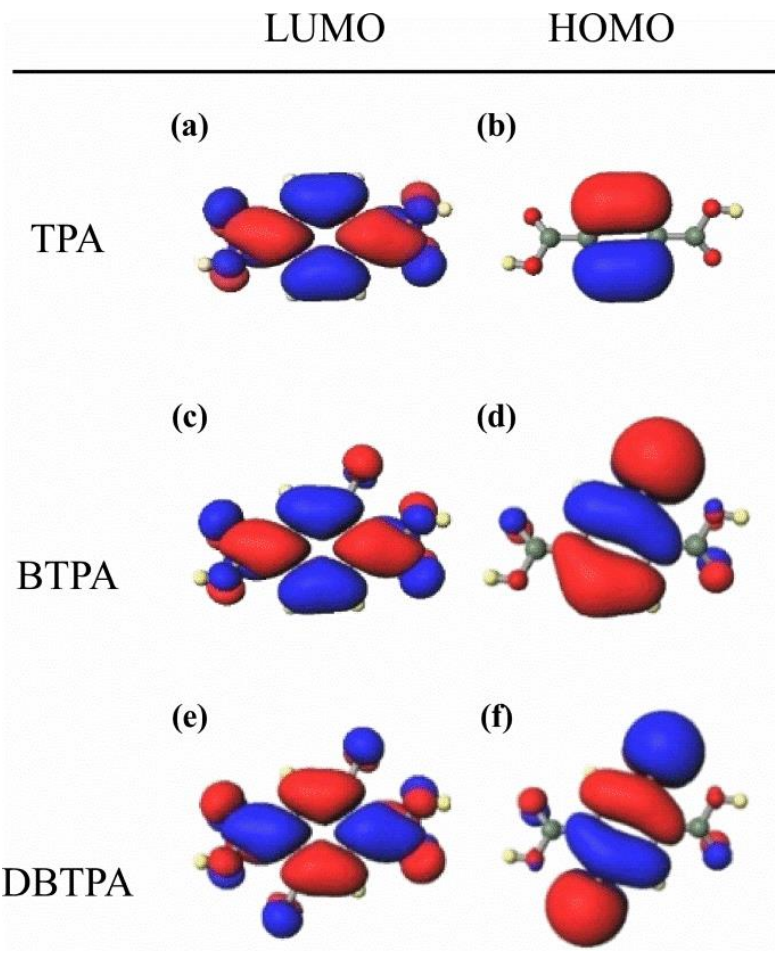

Figure 4. Calculated HOMOs and LUMOs. (a), (c) and (e) are LUMOs of TPA, BTPA and DBTPA. (b), (d) and (f) are HOMOs of TPA, BTPA and DBTPA.

In case of the BTPA monolayer, which has a bright spot for each molecule in negative bias STM image (Fig. 3(d)), structural analysis strongly indicate that the framework of the non-covalent interactions in the monolayer consists of two types of interactions: two halogen bonds between bromine and oxo groups (in the carboxyl group) $\left(\mathrm{Br}^{\cdots} \mathrm{O}=\mathrm{C}\right)$, bromine and hydroxyl groups (in the carboxyl group) ( $\mathrm{Br} \cdots \mathrm{OH})$ and hydrogen bonds between adjacent carboxyl groups (Figs. 5(b) and (e)). To prove the validity of the proposed model, electrostatic potentials of an isolated BTPA was also investigated by using DFT calculation, and molecular electrostatic potential maps of BTPA is shown in Fig. 6(a). Calculation showed that tips of bromine atoms have more positive electrostatic potential compared to equatorial areas around the bromine atoms. These polarizations are referred to as $\sigma$-hole and gives origin to halogen bonds. ${ }^{9-12}$ On the other hand, two oxygen atoms in carboxyl group have negative electrostatic potential. Thus, positive regions of bromine atoms are able to electrostatically interact with the negative part of the oxygen atoms in carboxyl groups of neighbouring molecules, indicating the halogen bonds formation of $\mathrm{Br}{ }^{\cdots} \mathrm{O}=\mathrm{C}$ and $\mathrm{Br}{ }^{\cdots} \mathrm{OH}$. It has been also reported that the $\mathrm{Br}^{\cdots} \mathrm{O}=\mathrm{C}$ and $\mathrm{Br}{ }^{\cdots} \mathrm{OH}$ halogen bonds play an important role in the association of $2 \mathrm{D}$ molecular assembly and biomolecules. ${ }^{28-30}$ These results strongly support the molecular packing structure of BTPA proposed by STM image (Figs. 5(b) and (e)). Unlike the TPA monolayer, the BTPA monolayer is characterized as a 1D noncovalent ladder-like network structure (Fig. 5(e)). Bromine atoms form halogen bonds with oxygen atoms in the carboxyl 


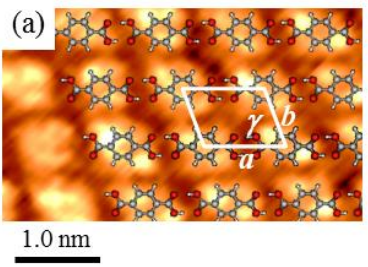

(d)

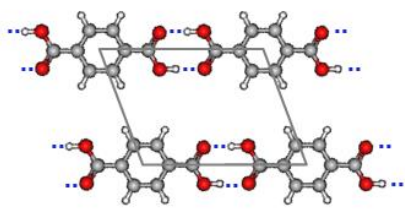

(b)

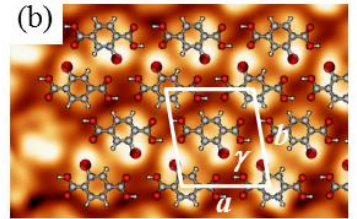

$1.0 \mathrm{~nm}$

(e)

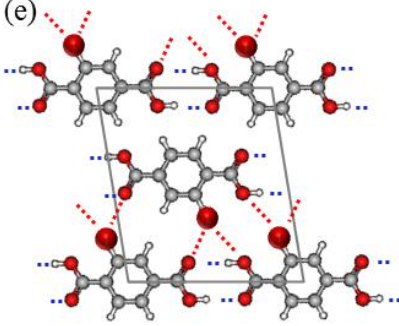

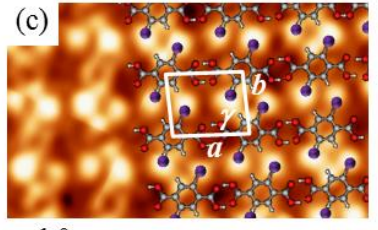

$1.0 \mathrm{~nm}$

(f)

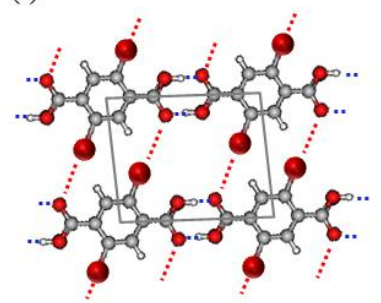

Figure 5. High resolution STM images of (a) TPA, (b) BTPA, and (c) DBTPA monolayers. Proposed molecular packing structures of (d) TPA, (e) BTPA, and (f) DBTPA monolayers. Blue and red dots line represent hydrogen and halogen bonds.

(a)

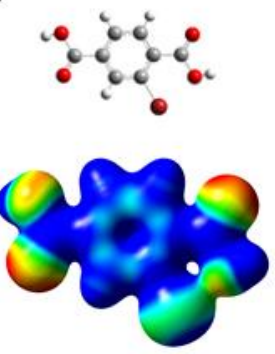

$-5.00 \times 10^{-2}$ au (b)
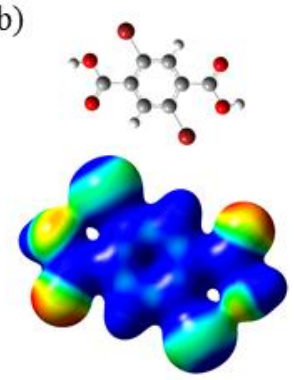

$8.00 \times 10^{-2} \mathrm{au}$

Figure 6. Calculated molecular electrostatic potential maps of (a) BTPA and (b) DBTPA.

\begin{tabular}{ccccc} 
& & TPA & BTPA & DBTPA \\
\hline \multirow{2}{*}{ Lattice } & $a$ & $0.96 \pm 0.05$ & $1.02 \pm 0.05$ & $1.07 \pm 0.05$ \\
parameter & $b$ & $0.89 \pm 0.05$ & $1.36 \pm 0.05$ & $0.87 \pm 0.05$ \\
& $\gamma$ & $70 \pm 5$ & $81 \pm 5$ & $82 \pm 5$ \\
\hline Network pattern & 1D chain-like 1D ladder-like & 2D lattice \\
\hline
\end{tabular}

Table 1. Proposed lattice parameters, type of interactions, and non-covalent network structures.

groups in adjacent molecule, contributing to the 1D ladder-like network formation with the hydrogen bond in the BTPA monolayer.

Structural analysis of the DBTPA monolayer was also performed to elucidate the framework of the non-covalently bonded structure. From the structural analysis, it is expected

that neighbouring DBTPA molecules interact with hydrogen and halogen bonds, and a 2D non-covalent lattice network structure is formed (Figs. 5(c) and (f)). Electrostatic potential of DBTPA is also shown in Fig. 6(b). As with BTPA molecule, there are the positive electrostatic potential at the tips of bromine atoms and the negative electrostatic potential at the regions of oxygen atoms for DBTPA. These calculation results support that two hydrogen bonds are formed between two adjacent carboxyl groups, and in addition, two bromine and oxo groups in the carboxyl groups are also bound by $\mathrm{Br}^{\cdots} \mathrm{O}=\mathrm{C}$ halogen bonds. Since $\mathrm{Br} \cdots \mathrm{O}=\mathrm{C}$ halogen bonds participate as intermolecular interaction, it is suggested that DBTPA molecules form the 2D non-covalent lattice network structure (Fig. 5(f)). BTPA and DBTPA monolayer analyses showed that each monolayer form different non-covalent network structure as shown Fig. 5(e) and (f). The difference strongly indicates that the addition of $\mathrm{Br}$ group in molecule tends to create new directional and halogen bonds with oxygen atoms, causing the significant structural change. To gain further insight of role of the halogen bond, it is also important to study control experiments using molecules replaced bromine groups with carboxyl groups. These investigations are now in progress.

The influence of halogen bonds between $\mathrm{Br}$ substituted molecules on 2D self-assembled monolayers was investigated, and it showed that $\mathrm{Br}{ }^{\cdots} \mathrm{Br}$ and $\mathrm{Br}^{\cdots} \mathrm{H}$ bonds play an important role in molecular assembly. ${ }^{17}$ Our results demonstrate that in the case of $\mathrm{Br}$ and $\mathrm{COOH}$ co-substituted molecules, $\mathrm{Br}$ atoms interact with $\mathrm{O}$ atoms in carboxyl group, and $\mathrm{Br}{ }^{\cdots} \mathrm{O}$ halogen and hydrogen bonds cooperatively assemble 2D molecular structure. The influence of coexistence of such halogen and hydrogen bonds upon 2D molecular structure formation has not been previously reported. The present findings provide new knowledge for designing novel 2D molecular structures.

\section{Conclusions}

The effect of hydrogen and halogen bonds on 2D molecular arrangements was investigated using STM and molecular orbital calculations. TPA, BTPA, and DBTPA molecules were assembled, and the monolayer arrangements were confirmed by STM. We found that there is strong bias polarity dependence of 
the STM images for BTPA and DBTPA monolayers. In the case of negative sample bias, unclear STM images were obtained, whereas positive sample bias gave clear molecular structures with relatively bright spots adjacent to the benzene rings. Molecular orbital calculations showed that the observed bright spots correspond to HOMO states of $\mathrm{Br}$ groups, allowing identification of the position of bromine groups in the molecule. Based on these results, the detailed packing structures were determined. In case of TPA, which is modified by carboxyl groups at the para positions of the benzene ring, a 1D linear non-covalent network structure was observed with two hydrogen bonds between adjacent carboxyl groups. However, unlike TPA, BTPA and DBTPA, which are modified by both carboxyl and bromine groups on the benzene ring, showed significantly different non-covalent network patterns.

Our results revealed that in the case of $\mathrm{Br}$ and $\mathrm{COOH}$ cosubstituted molecules, halogen and hydrogen bonds collaboratively create $2 \mathrm{D}$ molecular structures. These results suggest that the coexistence of various halogen and oxygen containing functional groups, such as hydroxyl, carbonyl, and carboxyl groups, in molecules would allow the precise design of desired 2D molecular structures. These findings will be of fundamental importance for material design and open up opportunities for tailoring novel 2D materials.

\section{Acknowledgements}

This work was partially supported by Japan Science and Technology Agency, PRESTO and the Grants-in-Aid for scientific research (nos. 22710127, 25109701, 25620003, and 24655162), from the Ministry of Education, Culture, Sports, Science, and Technology of Japan.

\section{Notes and references}

${ }^{a}$ Department of Chemistry, Faculty of Science, Hokkaido University, Sapporo, 060-0810, Japan. Fax: +81-(0)11-706-2704; Tel: +81-(0)11-7064810; E-mail: satoshi-yasuda@ sci.hokudai.ac.jp, kei@ sci.hokudai.ac.jp

b Japan Science and Technology Agency, Precursory Research for Embryonic Science and Technology, Kawaguchi, Saitama 332-0012, Japan.

1 J. A. A. W. Elemans,S. Lei, S. D. Feyter, Angew. Chem. Int. Ed. 2009, 48, 7298-7332.

2 M. Lackinger, S. Griessl, L. Kampschulte, F. Jamitzky, W. M. Heckl, Small 2005, 1, 532-539.

3 M. Lackinger, W. M. Heckl, Langmuir 2009, 25, 11307-11321.

4 S. Yasuda, H. Shigekawa, Jpn. J. Appl. Phys. 2003, 42, 4901-4904.

5 J. P. Rabe, S. Buchholz, Science 1991, 253, 424-427.

6 M. Lackinger, S. Griessl, T. Markert, F. Jamitzky, W. M. Heckl, J. Phys. Chem. B 2004, 108, 13652-13655.

7 M. O. Blunt, J. C. Russell, M. D. C. Giménez-López, J. P. Garrahan, X. Lin, M. Schröder, N. L. Champness, P. H. Beton, Science 2008, 322, 1077-1081.

8 M. Blunt, X. Lin, M. D. C. Gimenez-Lopez, M. Schröder, N. R. Champness, P. H. Beton, Chem. Commun., 2008, 2304-2306.

9 S. L. Price, A. J. Stone, J. Lucas, R. S. Rowland, A. E. Thornley, J. Am. Chem. Soc., 1994, 116, 4910-4918.

10 J. P. M. Lommerse, A. J. Stone, R. Taylor, F. H. Allen, J. Am. Chem. Soc., 1996, 118, 3108-3116.

11 P. Metrangold. H. Neukirch, T. Pilati, Acc. Chem. Res. 2005, 38, 386-395.
12 T. Clark, M. Hennemann, J. S. Murray, P.Politzer, J. Mol. Model., 2007, 13, 291-296.

13 P. Auffinger, F. A. Hays, E. Westhof, P. S. Ho, Proc. Natl. Acad. Sci. 2004, 101, 16789-16794.

14 P. Metrangolo, F. Meyer, T. Pilati, G. Resnati, G. Terraneo, Angew. Chem. Int. Ed. 2008, 47, 6114-6127.

15 G. A. Jeffrey, Introduction to Hydrogen Bonding; Oxford University Press: New York, 1997.

16 S. Zhu, C. Xing, W. Xu, G. Jin, Z. Li, Crys. Growth. Des., 2004, 4, 53-56.

17 R. Wilcken, M. O. Zimmermann, A. Lange, A. C. Joerger, F. M. Boeckler, J. Med. Chem., 2013, 56, 1363-1388.

18 M. M. S. Abdel-Mottaleb, G. Götz, P. Kilickiran, P. Bäuerle, E. Mena-Osteritz, Langmuir 2006, 22, 1443-1448.

19 D. Rohde, C.-J. Yan, L.-J. Wan, Langmuir 2006, 22, 4750-4757.

20 Q. Chen, T. Chen, D. Wang, H.-B. Liu, Y.-L. Li and L.-J. Wan, Proc. Natl. Acad. Sci., 2010, 107, 2769-2774.

21 R. Gutzler, O. Ivasenko, C. Fu, J. L. Brusso, F. Rosei, D. F. Perepichka, Chem. Comm., 2011, 47, 9453-9455.

22 H. Bertrand, F. Silly, M.-P. T.-Fichou, L. Tortech, D. Fichou, Chem. Comm., 2011, 47, 10091-10093.

23 R. Gutzler, C. Fu, A. Dadvand, Y. Hua, J. M. MacLeod, F. Rosei and D. F. Perepichka, Nanoscale, 2012, 4, 5965-5971.

24 F. Silly, J. Phys. Chem. C, 2013, 117, 20224-20249.

25 B. Zha, X. Miao, P. Liu, Y. Wu and W. Deng, Chem. Commun., 2014, 50, 9003-9006.

26 R. Gutzler, O. Ivasenko, C. Fu, J. L. Brusso, F. Rosei and D. F. Perepichka, Chem. Commun., 2014, 50, 5714-5716.

25 D. M. Cyr, B. Venkataraman, G. W. Flynn,; A. Black, G. M. Whitesides, J. Phys. Chem. 1996, 100, 13747-13759.

26 H. S. Lee, S. Iyengar, I. H. Musselman, Langmuir 1998, 14, 74757483.

27 F. Stevens, D. J. Dyer, U. Müller, D. M. Walba, Langmuir 1996, 12, 5625-5629.

28 L. A. Hardegger, B. Kuhn, B. Spinnler, L. Anselm, R. Ecabert, M. Stihle, B. Gsell, R. Thoma, J. Diez, J. Benz, J-M. Plancher, G. Hartmann, D. W. Banner, W. Haap, F. Diederich, Angew. Chem. Int. Ed. 2011, 50, 314-318.

29 J. K. Yoon, W.-J. Son, K.-H, Chung, H. Kim, S. Han, S.-J. Kahng, J. Phys. Chem. C, 2011, 115, 2297-2301.

30 E. I. Howard, R. Sanishvili, R. E. Cachau, A. Mitschler, B. Chevrier, P. Barth, V. Lamour, M. V. Zandt, E. Sibley, C. Bon, D. Moras, T. R. Schneider, A. Joachimiak, A. Podjarny, Structure, Function, and Bioinformatics 2004, 55, 792-804. 